\title{
Clinical applications of comparative genomic hybridization
}

Brynn Levy, MSc, Teresa M. Dunn, PhD, Sara Kaffe, MD, Nataline Kardon, MD, and Kurt Hirschhorn, MD

Purpose: Comparative genomic hybridization (CGH) is a powerful DNA-based cytogenetic technique that allows the entire genome to be scanned for chromosomal imbalances without requiring the sample material to be mitotically active. During the past 2 years we received many requests from various medical centers around the country to use CGH to resolve the identity of aberrant chromosomal material. Methods: We report the use of $\mathrm{CGH}$ for the evaluation of 12 clinical postnatal cases in which traditional cytogenetic analysis yielded ambiguous results. This series consisted of five marker chromosomes, five unbalanced transloca tions, and two intrachromosomal duplications. Results: Identification and characterization of the additional unknown chromosomal material was achieved with use of CGH. All CGH findings were validated by traditional fluorescence in situ hybridization and other specialized staining techniques. Conclusions: These results demonstrate the effective use of $\mathrm{CGH}$ as a focused, single-step method for the identification of chromosomal material of unknown origin.

Key words: $C G H, F I S H$, marker chromosomes, intrachromosomal duplications, unbalanced translocations

\section{INTRODUCTION}

From the Department of Human Genetics and Pediatrics, Mount Sinai School of Medicine, New York; and the Departinent of Pediatrics, Mount Sinai Services at Elmhurst Hospital Center. Elmhurst, New York

Address correspondence to: Brynn Levy. MSi (Med), Department of Human Genetics, Box 1497, Mount Sinai School of Medicine, One Gustave L. Levy Place, New York, NY 10029. E-mail:Blevy@snttplink.mssm.edu

01998 Gentetics in Medicine. All rights reserved. $1098-3600 \$ 0.00$
Approximately $3 \%$ to $4 \%$ of liveborn babies have a major congenital defect. ${ }^{1}$ These defects include unbalanced chromosome abnormalities which occur in approximately 1 in 250 newborns. ${ }^{2}$ Between $15 \%$ and $20 \%$ of all pregnancies end in a spontaneous abortion, and of these approximately $50 \%$ are associated with chromosome abnormalities. ${ }^{2}$ A precise diagnosis in a newborn or prenatal sample with a chromosome abnormality is critical for appropriate genetic counseling as well as for the clinical management of an infant. It also provides the parents with a realistic prognosis. There are many instances when cytogenetic analysis is unsuccessful in producing a result because the specimen cannot be cultured. There are also many occasions when extrachromosomal material remains unidentifiable even after numerous standard cytogenetic staining methods have been attempted. Molecular cytogenetic techniques, such as fluorescence in situ hybridization (FISH), have brought a new depth to clinical cytogenetics by facilitating the identification of chromosomal material of unknown origin. This approach often requires using multiple whole chromosome paints (WCP) until the source chromosome is identified..$^{3-5}$ Alternative approaches include reverse FISH, using probes derived from the microdissected chromosome region of interest ${ }^{6-8}$; and multicolor FISH probing. ${ }^{9,10}$ However, these techniques require specialized equipment in addition to the regular cytogenetic/FISH image analysis equipment that now is commonly found in many comprehensive cytogenetic laboratories. Although multicolor FISH can determine the origin of the unknown material, it does not identify the specific location or breakpoints on the chromosome from which the extra material originated.

Comparative genomic hybridization (CGH) is a relatively new molecular cytogenetic technique that allows for the identification of chromosomal gains or losses by scanning the entire genome in a single step. It has the distinct advantage of providing a genome-wide search without any prior information about the chromosomal aberration in question. It is accomplished by in situ hybridization of differentially labeled total genomic specimen DNA and normal reference DNA to normal human metaphase chromosome spreads. ${ }^{11-14}$ Hybridization of the specimen and reference DNA can be distinguished by their different fluorescent colors. The relative amounts of specimen and reference DNA hybridized at a particular chromosome position are contingent on the relative excess of those sequences in the two DNA samples and can be quantified by calculation of the ratio of their different fluorescent colors. ${ }^{11-14}$ Specimen DNA is traditionally labeled with a green fluorochrome such as fluorescein isothiocyanate and the normal reference DNA with a red fluorochrome like Texas Red. A gain of chromosomal material in a specimen would be detected by an elevated greento-red ratio, whereas deletions or chromosomal losses would produce a reduced green-to-red ratio. ${ }^{11-14}$ $\mathrm{CGH}$ analysis provides information about the origin of gains and losses of chromosomal material and maps these imbalances to their position on the source chromosome. Although CGH cannot detect balanced chromosomal rearrangements, in clinical cytogenetics phenotypically abnormal patients usually have unbalanced chromosomal abnormalities. CGH has been used primarily in cancer cytogenetic studies, and data on its utility in a clinical genetics setting are limited. ${ }^{15-22}$ Because CGH analysis is DNA-based, culturing the specimen and relying on the availability and quality of metaphase spreads are not considerations. Thus, even nonviable tissues are amenable to analysis by CGH. These factors make CGH an attractive technique for application in clinical cytogenetic analysis, especially for samples in which a 
complete and detailed karyotype cannot be obtained by conventional methods.

This report describes a series of postnatal clinical cases in which conventional cytogenetic studies revealed karyotypes with additional chromosomal material of uncertain origin. In all instances, CGH was used effectively to identify the origin of the unknown chromosomal material. FISH with chromosome-specific paints and probes as well as specialized cytogenetic staining techniques were then used to confirm the $\mathrm{CGH}$ results.

\section{MATERIALS AND METHODS \\ Patients}

The clinical information described below was provided by the referring institutions.

Case 1: Eleven-year-old female, short stature (49 inches), Tanner 1 , and normal IQ. Initial karyotype, $47, \mathrm{XX}$, + mar. Normal parental chromosomes. Referred by Shari Fallet and Joyce E. Fox from the Long Island Jewish Medical Center (New Hyde Park, NY).

Case 2: Twenty-six-year-old male, profound mental retardation, prominent ears, short stature ( 62 inches), choreiform movements, has few words, follows simple commands, and has normal adult male genitalia. Initial karyotype, $47, \mathrm{XY}$, + mar. Normal parental chromosomes. Referred by Ernest Lieber and Gamil Kostandy from the New York Methodist Hospital (Brooklyn, NY).

Case 3: Two-year-old male, diagnosed clinically with Prader-Willi syndrome later demonstrated to be caused by maternal uniparental heterodisomy, normal birth weight, hypotonic, and severe developmental delay. Initial karyotype, 47,XY, + mar. Normal maternal chromosomes, paternal cytogenetic studies not done. Referred by Nancy J. Carpenter from the H.A. Chapman Institute of Medical Genetics (Tulsa, OK).

Case 4: Twenty-month-old male, birth weight $6 \mathrm{lbs}$, $3 \mathrm{oz}$, anal hypoplasia at birth, severe developmental delay, hypotonia, one seizure in infancy with normal MRI and EEG, small ears with thick lobes and abnormal architecture, small pointed chin, contractures of thumbs, increased deep tendon reflexes, and bilateral Babinsky. Initial karyotype, 47,XY, + mar. Normal maternal chromosomes, father unavailable for cytogenetic analysis. Referred from our own institution.

Case 5: Fifteen-year-old male, arthrogryposis, scoliosis, mild cognitive delay, bilateral syndactyly of digits 2, 3, and 4, bilateral club feet, short arms, peripheral pulmonic stenosis, short stature ( $<5$ th percentile), head circumference at the 5th percentile, prominent jaw, everted lower lip, down-slanting palpebral fissures, ptosis, mild epicanthal folds, small low-set cupped ears with missing lobes and bilateral sinus tracks with a left ear pit, very high arched palate, deep central crease in the tongue, very long fingers and hands ( $>97$ th percentile), ulnar deviation of hands, flexed elbows with mild pterygia, bilateral overlap of second and third toes, and abnormally long first toes. Initial karyotype, $46, \mathrm{XY} / 47, \mathrm{XY}+\mathrm{mar}$ ( $50 \%$ mosaicism). Normal parental chromosomes. Referred by Elaine $\mathrm{H}$.
Zackai from the Children's Hospital of Philadelphia (Philadelphia, PA).

Case 6: Two-year-old male, bitemporal depression, elevated nasal bridge, midface hypoplasia, long philtrum, low-set ears, micropenis, undescended testes, and swallowing dysfunction requiring tracheostomy and GE tube. Initial karyotype, 46,XY,add(10q). Normal parental chromosomes. Referred by Nancy J. Carpenter from the H.A. Chapman Institute of Medical Genetics (Tulsa, OK).

Case 7: Ten-year-old male, severe mental retardation, elongated face, bitemporal narrowing, prominent glabella, short palpebral fissures, and bifid uvula. Initial karyotype, 46,XY,add(lq). Normal maternal chromosomes, father unavailable for cytogenetic analysis. Referred from our own institution.

Case 8: Eight-year-old female, developmental delay, short stature (45 inches), normal head circumference but prominent high frontal region, myopia, and sacral dimple. Initial karyotype, $46, \mathrm{XX}$,add $(\mathrm{Xp})$. Normal parental chromosomes. Referred by Lawrence R. Shapiro and Monica Zak from the Westchester County Medical Center (Valhalla, NY).

Case 9: Nine-year-old female, birth weight $1800 \mathrm{~g}$, developmental delay, cleft palate, possible congenital heart disease, horseshoe kidney, short stature, and short stubby fingers. Initial karyotype, $46, \mathrm{XX},-\mathrm{X},+\operatorname{der}(\mathrm{X}) \mathrm{t}(\mathrm{X} ;$;?)(p22.1;?). Normal paternal chromosomes. The mother carries an X/19 translocation, a fact that was not known when the CGH studies were done. Referred from our own institution.

Case 10: Two-week-old male, 37 weeks gestation, birth weight $2025 \mathrm{~g}, 2$ vessel cord, conotruncal malalignment, microcephalic ( $<10$ th percentile), short ( $<15$ th percentile), small palpebral fissures, epicanthal folds, broad forehead, malformed ears, hypertelorism, prominent nasal bridge, upturned nose, long philtrum, thin upper lip, down-turned mouth, midface hypoplasia, micrognathia, tapering fingers with hypoplastic nails, webbing between digits, small penis and scrotum, and bilateral inguinal hernias. Initial karyotype, 46 , XY,add(21p). Normal parental chromosomes. Referred from our own institution.

Case 11: Nine-year-old female, markedly delayed expressive language skills, microcephalic $(<5$ th percentile), 5 th percentile for height and 25 th percentile for weight, down-slanted palpebral fissures, hypertelorism, low-set ears, preauricular pits, malar hypoplasia, long smooth philtrum, micrognathia, brachydactyly, bilateral short fourth phalanx and clinodactyly of the fifth finger, left hand polydactyly, left foot with six toes with partial syndactyly of the fifth and sixth toes, enlarged liver and spleen, primary pulmonary hypertension, and high arched palate. Initial karyotype, $46, X X$,add (16p). Normal parental chromosomes. Referred from our own institution.

Case 12: Four-year-old female, severe developmental delay, partial agenesis of the corpus callosum, abnormal cortex, seizure disorder, weight and head circumference normal at birth, poor 
suck and hypotonia at birth, bilateral simian crease, bilateral syndactyly of fourth and fifth toes, microcephalic ( $<5$ th percentile), epicanthal folds, thickened ear creases, no spoken words, good comprehension, and wide-based unsteady gait. Initial karyotype, 46,XX,add(13q). Cytogenetic analysis of parents not performed. Referred by Elaine $H$. Zackai from the Children's Hospital of Philadelphia (Philadelphia, PA).

\section{Comparative genomic hybridization}

CGH was performed according to the method of Kallioniemi et al., ${ }^{14}$ with the following modifications. DNA from peripheral blood lymphocytes of each patient and a karyotypically normal control (sex matched to the patient) was isolated by standard techniques. ${ }^{23}$ Reference and sample DNA were labeled by nick translation with Texas Red-5-deoxyuridine triphosphate (dUTP) and fluorescein-12-dUTP (DuPont NEN, Boston, MA), respectively. The hybridization mixture consisted of $200 \mathrm{ng}$ specimen DNA, 200 ng reference DNA, and $20 \mu \mathrm{g}$ Cot-1 DNA (GIBCO/BRL, Gaithersburg, MD) in $10 \mu \mathrm{L}$ of Hybrisol VII (Oncor, Gaithersburg, MD). The probe mixture was hybridized to normal male metaphase spreads $(46, \mathrm{XY})$ for 3 days at $37^{\circ} \mathrm{C}$. Unbound DNA fragments were removed by washing in $2 \times$ saline sodium citrate (SSC) at $72^{\circ} \mathrm{C}$ for 5 minutes, $4 \times$ SSC at $37^{\circ} \mathrm{C}$ for 5 minutes, $4 \times$ SSC, $0.1 \%$ Triton $\mathrm{X}$ at $37^{\circ} \mathrm{C}$ for 5 minutes, $4 \times \mathrm{SSC}$ at $37^{\circ} \mathrm{C}$ for 5 minutes, $2 \times$ SSC at room temperature for 5 minutes, and in $\mathrm{H}_{2} \mathrm{O}$ at room temperature for 5 minutes.

CGH images were captured with an ImagePoint cooled CCD video camera (Photometrics, Tucson, AZ) through a Labophot-2A fluorescence microscope (Nikon, Melville, NY). Chromosome identification and karyotyping was facilitated by counterstaining with $0.1 \mu \mathrm{g} / \mu \mathrm{L}$ 4,6-diamidino-2-phenylindole (DAPI) in Vectashield (Vector Laboratories, Burlingame, CA) ${ }^{24}$ Metaphases with relatively straight nonoverlapping chromosomes were analyzed. The fluorescence ratios (green/red) for at least 10 of each autosome and 7 of each sex chromosome were obtained per slide. Background fluorescence was subtracted, and the green-to-red ratio of each entire metaphase was normalized to 1.0. Data from several metaphase spreads were combined to generate an average ratio profile for each chromosome. The lines in the CGH profiles (Fig. 1: column B) represent ratios of (from left to right) $0.5,0.75,1.0,1.25$, $1.5,1.75$, and 2.0. A ratio of 1.0 represents the balanced state of the chromosomal copy number. An upper threshold of $>1.20$ was used to define a gain of chromosomal material, whereas a lower threshold of $<0.80$ was used to interpret a loss (deletion) of chromosomal material. ${ }^{25}$ Gains and losses were only considered relevant if the $95 \%$ confidence intervals (data not shown), derived from the combination of each single chromosome profile, met the threshold criteria. Digital image analysis was performed with a Cytovision Probe system and CGH software (Applied Imaging Corp, Santa Clara, CA).

\section{Fluorescence in situ hybridization}

Whole chromosome paints were purchased either from Vysis (Downers Grove, IL) or from Oncor (Gaithersburg, MD). Chromosome arm-specific paints were purchased from ALTechnologies (Arlington, VA), and locus-specific probes were obtained from Oncor (Gaithersburg, MD). FISH studies were performed according to the manufacturer's instructions. Metaphase spreads were counterstained with

Table 1

Summary of the cytogenetic, CGH, and FISH/DA-DAPI results of 12 clinical postnatal cases

\begin{tabular}{|c|c|c|c|c|}
\hline Case & Initial Karyotype & CGH Result & FISH/DA-DAPI Result & Revised Karyoptye \\
\hline 1 & $47, \mathrm{XX},+$ mar & enh(15)(pter-q11.2) & $\operatorname{mar}(\mathrm{DA}-\mathrm{DAPI}+)$ & $46, \mathrm{XX},+$ mar.rev ish enh(15)(pterq11.2) \\
\hline 2 & $47, X Y,+\operatorname{mar}$ & $\operatorname{enh}(15)$ (pter-q14) & $\operatorname{mar}(\mathrm{DA}-\mathrm{DAPI}+)$ & 46,XY,+mar.rev ish enh(15)(pterq14) \\
\hline 3 & $47, \mathrm{XY},+\operatorname{mar}$ & enh(15)(pter-q12) & $\operatorname{mar}($ wcp $15+)$ & $46, \mathrm{XY},+$ mar.rev ish enh(15)(pterq12) \\
\hline 4 & $47, \mathrm{XY},+\mathrm{mar}$ & $\operatorname{enh}(18 p)$ & $\operatorname{mar}(w c p 18+)$ & $46, X Y,+$ mar.rev ish enh(18p) \\
\hline 5 & $\begin{array}{l}46, X Y / 47, X Y+m a r \\
(50 \% \text { Mosaicism) }\end{array}$ & $\operatorname{enh}(1)(p 13-q 12)$ & $\operatorname{mar}(w c p l+)$ & $46, \mathrm{XY} / 47, \mathrm{XY}+$ mar.rev ish enh(1)(p13q12) \\
\hline 6 & $46, X Y, \operatorname{add}(10 q)$ & $\begin{array}{l}\operatorname{enh}(7)(q 31.3 \text {-qter }) \\
\operatorname{dim}(10)(q 26-q \text { ter })\end{array}$ & $\operatorname{add}(10 q)(w c p 7+)$ & $\begin{array}{l}\text { 46,XY,add }(10)(\mathrm{q} 26) \cdot \mathrm{rev} \text { ish } \operatorname{der}(10) \mathrm{t}(7 ; 10) \\
(\mathrm{q} 31.3 ; \mathrm{q} 26) \operatorname{enh}(7)(\mathrm{q} 31.3 \mathrm{qter}) \operatorname{dim}(10)(\mathrm{q} 26 \mathrm{qter})\end{array}$ \\
\hline 7 & $46, X Y$,add (lq) & $\operatorname{enh}(8)(q 23-q \operatorname{ter})$ & $\operatorname{add}(\operatorname{lq})(w c p 8+)$ & $\begin{array}{l}\text { 46,XY,add(1)(q44).rev ish } \operatorname{der}(1) t(1 ; 8) \\
(q 44 ; q 23) \text { enh }(8)(q 23 q \text { ter })\end{array}$ \\
\hline 8 & $46, X X, \operatorname{add}(X p)$ & enh(10)(pter-p12.33) & $\operatorname{add}(X p)(w c p 10+)$ & $\begin{array}{l}\text { 46,XX,add }(X)(p 22.3) \cdot r e v \text { ish } \operatorname{der}(X) t(X ; 10) \\
(p 22.3 ; p 12.33) \text { enh(10)(pterp12.33) }\end{array}$ \\
\hline 9 & $\begin{array}{l}46, X X,-X,+\operatorname{der}(X) \\
t(X ; ?)(p 22.1 ; ?)\end{array}$ & $\begin{array}{l}\operatorname{enh}(19)(q 13.1-q \operatorname{ter}) \\
\operatorname{dim}(X)(\text { pter-p22.13) }\end{array}$ & $\operatorname{der}(X p)(w c p 19+; D X Z 1-)$ & $\begin{array}{l}\text { 46,X,-X+der(X).rev ish } \operatorname{der}(X) t(X ; 19) \\
\text { (p22.13;q13.1)mat enh(19)(q13.1qter)dim(X) } \\
\text { (pterp22.13) }\end{array}$ \\
\hline 10 & $46, X Y$, add $(2 l p)$ & $\operatorname{enh}(17 p)$ & $\operatorname{add}(21 \mathrm{p})($ wcp $17+; \mathrm{D} 17 \mathrm{~S} 379+)$ & $\begin{array}{l}\text { 46,XY,add(21)(p11.1).rev ish } \operatorname{der}(21) t(17 ; 21) \\
\quad(\mathrm{p} 11.1 ; \mathrm{p} 11.1) \operatorname{enh}(17)(\mathrm{pterp} 11.1)\end{array}$ \\
\hline 11 & $46, X X, \operatorname{add}(16 p)$ & $\operatorname{enh}(16)(q 12.1-q 22.1)$ & $\operatorname{add}(16 \mathrm{p})(\mathrm{pcp} 16 \mathrm{p}+; \mathrm{pcp} 16 \mathrm{q}++)$ & $\begin{array}{l}\text { 46,XX, add(16p). ish dup inv ins } \\
(16)(\mathrm{p} 11.2 \mathrm{q} 22.1 \mathrm{q} 12.1)(\mathrm{pcp} 16 \mathrm{p}+\text {;pcp16q++).rev } \\
\text { ish enh(16)(q12.1q22.1) }\end{array}$ \\
\hline 12 & $46, \mathrm{XXadd}(13 q)$ & $\operatorname{enh}(13)(q 31-q 33)$ & $\begin{array}{l}\operatorname{add}(13 q)(B A C 13 C 5+; \\
\text { BAC13C } 7++; B A C 13 C 11++)\end{array}$ & $\begin{array}{l}\text { 46,XX,add(13q).ish dup inv ins } \\
(\mathrm{q} 31.3 \mathrm{q} 33.1 \mathrm{q} 31.3)(\mathrm{BAC} 13 \mathrm{C} 5+, \mathrm{BAC} 13 \mathrm{C} 7++ \\
\mathrm{BAC} 13 \mathrm{C} 11++) . r e v \text { ish enh(13)(q31.3q33.1) }\end{array}$ \\
\hline
\end{tabular}




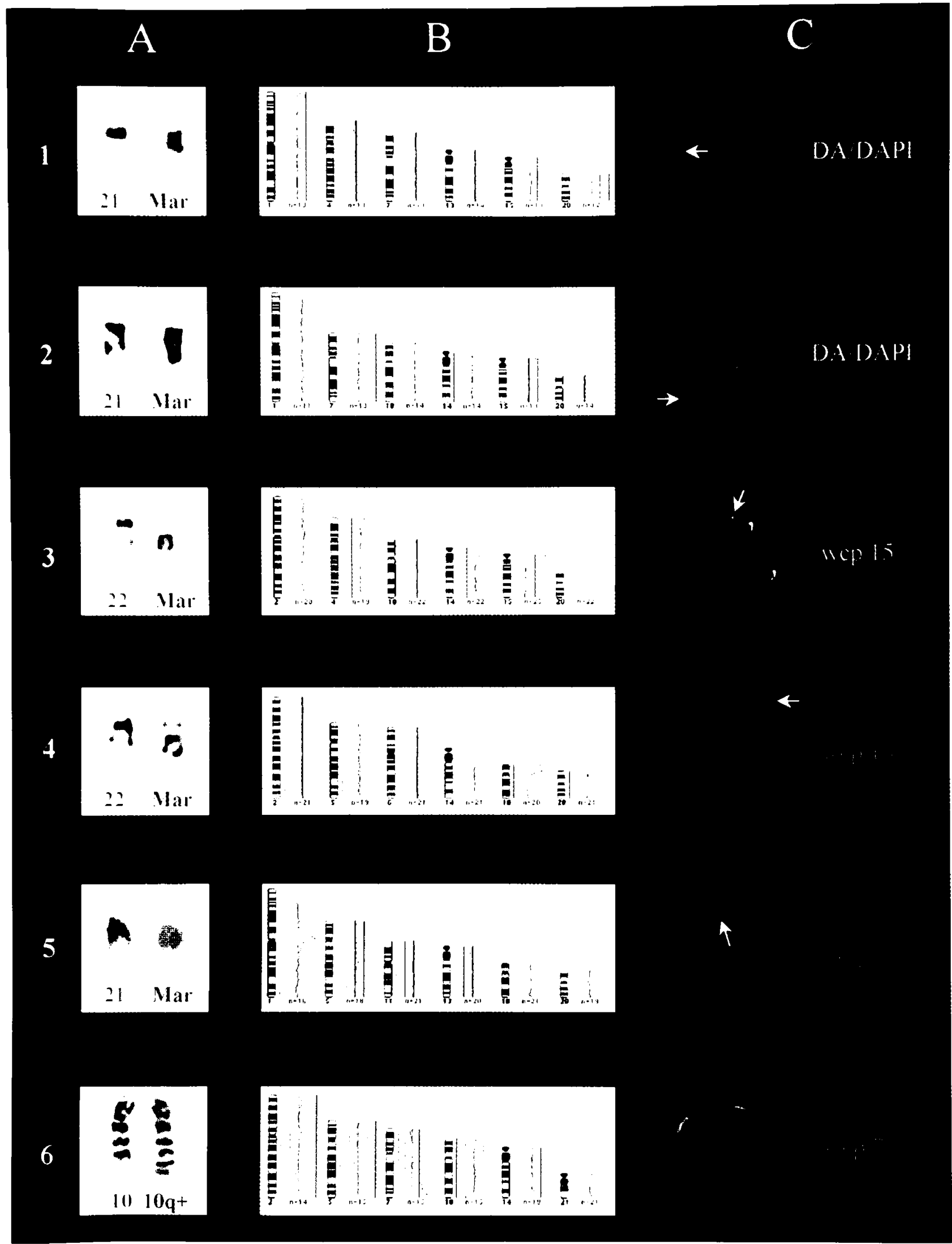

Fig. 1 Comprehensive cytogenetic workup of 12 clinical postnatal cases. (A) Partial karyotypes showing the G-banded cytogenetic aberration (labeled in red). Marker chromosomes are paired with normal chromosomes 21 or 22 (labeled in blue) for an indication of size. The remaining abnormal chromosomes are paired with their normal counterpart (labeled in blue). (B) Partial ideograms and CGH profiles indicating the source of the additional chromosomal material. CGH results are summarized in Table 1 with additional details in the results section. (See Materials and Methods sections for interpretation of CGH results.) (C) Confirmation of CGH findings by FISH or DA-DAPI staining. FISH labels are color-coded to match figure. WCP, whole chromosome paint; $\mathrm{CGH}$, comparative genomic hybridization; FISH, fluorescence in situ hybridization. 


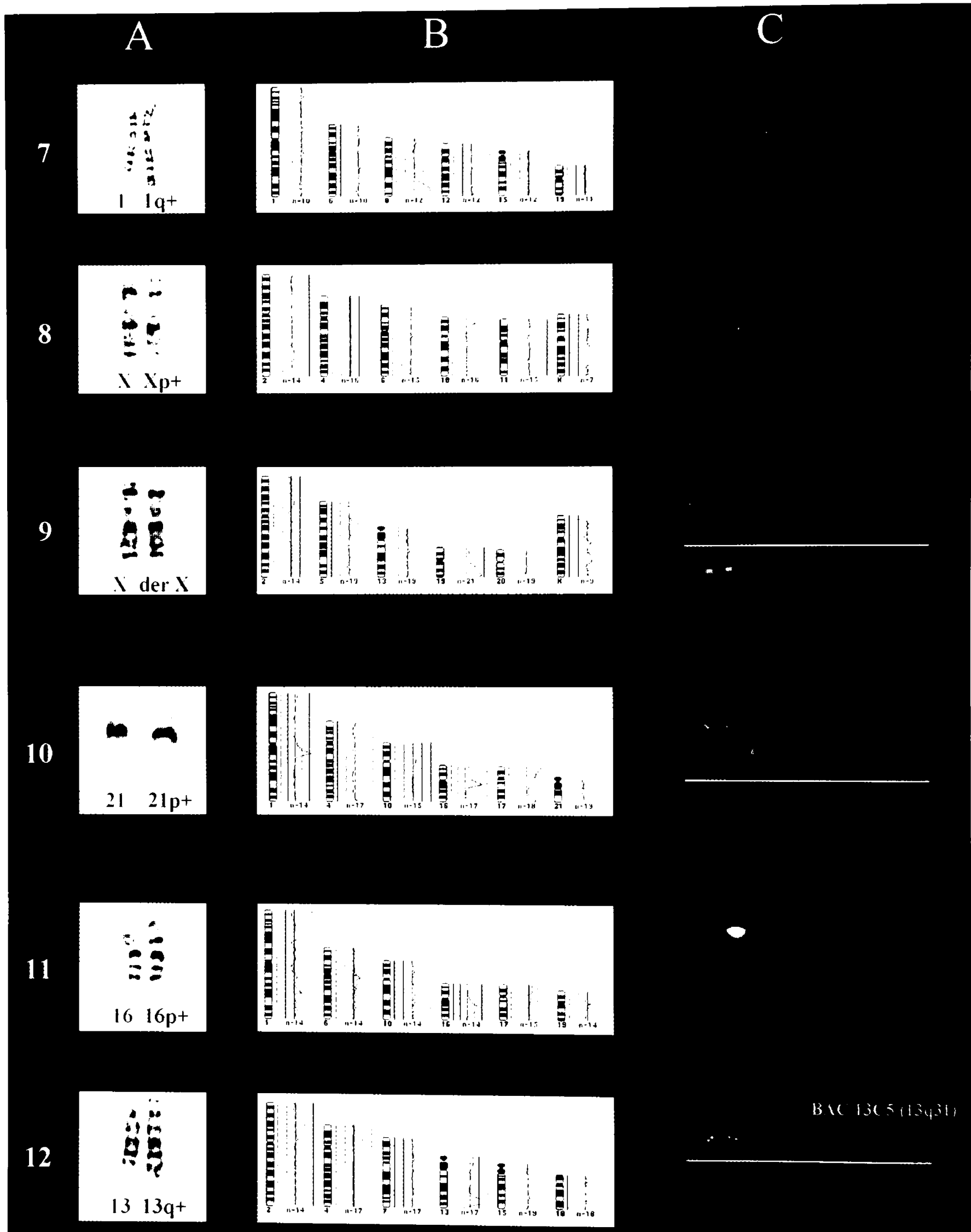


$0.1 \mu \mathrm{g} / \mu \mathrm{L}$ DAPI in Vectashield (Vector Laboratories, Burlingame, CA). ${ }^{24}$

Bacterial artificial chromosome (BAC) probes were obtained from Xiao-Ning Chen and Julie R. Korenberg (Mapped BAC Resource, Cedars-Sinai Medical Center, CA) and were labeled by nick translation with either digoxigenin-11-dUTP or biotin16-dUTP (Boehringer Manheim, Indianapolis, IN). The hybridization mixture consisted of $150 \mathrm{ng}$ DNA of each probe, $4 \mu \mathrm{g}$ Cot-l DNA, and $7 \mu \mathrm{g}$ herring sperm DNA (GIBCO/BRL, Gaithersburg, MD) in $10 \mu \mathrm{L}$ of Hybrisol VII (Oncor, Gaithersburg, MD). The probe mixture was hybridized overnight in a humid chamber at $37^{\circ} \mathrm{C}$ to metaphase spreads derived from the patient in case 12 . Unbound DNA fragments were removed by washing in $2 \times \mathrm{SSC}$ at $72^{\circ} \mathrm{C}$ for 5 minutes and $1 \times$ phosphate-buffered detergent (PBD) for 3 minutes at room temperature. Slides were then incubated with $60 \mu \mathrm{L}$ Avidin-FTC/ antidigoxigenin-rhodamine (Oncor, Gaithersburg, MD) for 15 minutes at $37^{\circ} \mathrm{C}$. This was followed by three 3-minute washes in $1 \times \mathrm{PBD}$ at room temperature. Metaphase spreads were counterstained with $0.1 \mu \mathrm{g} / \mu \mathrm{L}$ DAPI in Vectashield (Vector Laboratories, Burlingame, CA). ${ }^{24} \mathrm{FISH}$ images were captured as described in the CGH section.

\section{RESULTS}

From October 1996 to April 1998, CGH analysis was performed on seven postnatal clinical samples received from six different medical centers around the country. Five additional samples were referred from our own institution. All samples were derived from patients in whom traditional cytogenetic analyses revealed extrachromosomal material of unknown origin. In all instances, CGH analysis accurately identified the derivation of the aberrant chromosome. All CGH findings were confirmed either by FISH or DA-DAPI staining. A summary of the results of the comprehensive workup of each patient is shown in Table 1. Figure 1 shows the cytogenetic aberrations in question (column A), the CGH ratio profiles (column B), and the confirmation of the CGH results (column C). CGH results are displayed with representative chromosome profiles. All other chromosomes (data not shown) had normal CGH profiles, with no significant deviation from a value of 1.0 .

\section{Markers}

The first five cases demonstrate the effective use of CGH in resolving the identity of marker chromosomes. The sizes of the markers varied considerably (Fig. 1: 1A-5A). The markers that were derived from chromosome 15 probably were formed by an inversion duplication event in cases 1,2 , and possibly in case 3, as judged by their G-bands by trypsin using Giemsa (GTG) pattern. Concurrent molecular and molecular-cytogenetic study by the referring institution of the patient in case 3 did not demonstrate the presence of the Prader-Willi probes (SNRPN and D15S11) in the marker chromosome but revealed uniparental maternal heterodisomy of chromosome 15. The clinical features presenting in this patient are therefore considered to result from an initial trisomy 15 event with subsequent loss of the majority of the paternal 15 chromosome leaving two maternal chromosomes 15 and a paternally derived marker chromosome 15.
The marker in case 4 showed an overrepresentation of the entire short arm of chromosome 18 (Fig. 1: 4B). Together with the cytogenetic appearance (Fig. 1: 4A), this marker was determined to be an $18 \mathrm{p}$ isochromosome. In case 5 , an overrepresentation of patient DNA at the centromeric region of chromosome 1 (Fig. 1: 5B) was considered to be a significant and real finding compared with the frequently observed increase in this region which is primarily a result of the repetitive nature of the heterochromatic DNA. A comparison of the chromosome 1 ratio profile in cases 5 and 10 (Fig. 1: 5B vs. 10B) reveals an upward shift into the short arm in case 5 when matched with the insignificant pattern in case 10. The extrachromosomal material therefore derives from proximal $1 p$ and includes some of the heterochromatic region of proximal 1q.

\section{Translocations}

The designation of the source of the additional material in cases 6 to 10 was achieved easily by CGH. These five cases represent unbalanced translocations. The translocations in cases 6,8 , and 10 were de novo in nature because cytogenetic analyses of the parents revealed normal karyotypes. Chromosome analysis of the mother in case 7 revealed a normal karyotype; the father was not available for cytogenetic studies. In case 9 , chromosome analysis of the parents, after the CGH studies, revealed that the mother carried a balanced X/19 translocation. Dual color FISH in cases 6 to 9 readily confirmed the origins of the additional material in the unbalanced chromosome (Fig. 1: C6-C9). In addition to a gain of chromosome $19 q$ material in case 9 , a loss in the short arm of the $\mathrm{X}$ chromosome is also evident from the CGH profile (Fig. 1: 9B). This loss is also discernible from the GTG image (Fig. 1:9A). The deletion of the distal area of Xp in case 9 was confirmed using a locus-specific probe (STS gene probe) which showed no signal on the derivative $\mathrm{X}$ chromosome (Fig. 1: 9C). In case 10, the CGH profile indicated that the short arm of chromosome 17 was the source of the additional material on the $21 \mathrm{p}+$ chromosome. Verification was obtained by FISH using a chromosome 17 WCP and a locus-specific probe (MillerDieker gene probe) for the short arm of chromosome 17. Both showed a signal on derivative chromosome 21 as well as on the two normal chromosomes 17 . The apparent overrepresentation of the distal region of $17 \mathrm{q}$ (Fig. $1: 10 \mathrm{~B}$ ) in case 10 was not significant at the $95 \%$ confidence interval level, as determined by the confidence intervals produced by combining the CGH ratios from 18 representatives of chromosome 17 (data not shown).

\section{Intrachromosomal duplications}

The final two cases represent examples of the utility of CGH in identifying and elucidating intrachromosomal duplications. In case 11 , the CGH ratio profile for chromosome 16 , indicating a gain of material (Fig. 1: 11B), is distinctly different from the inconsequential gain expected for the heterochromatic region of chromosome 16 (Fig. 1: 10B vs. 11B). Using dual color arm specific paints for chromosome 16 clearly shows the insertion of $16 \mathrm{q}$ material into the short arm of chromosome 16 (Fig. 1: 11C). The orientation of the insertion (q12.1q22.1) seems to be inverted from the GTG appearance; however, further molecular cytogenetic analysis 
is needed for verification. In case 12 (Fig. 1: 12B), an intrachromosomal duplication involving the long arm of chromosome 13 clearly can be inferred from the $\mathrm{CGH}$ profile. The duplication was confirmed using probes specific for bands $13 q 31$ (BAC 13C5), $13 \mathrm{q} 32$ (BAC 13C7), and 13q32/33 (BAC 13C11) which showed one signal for $B A C 13 C 5$ and two signals each for BACs $13 C 7$ and 13C11 (Fig. 1: 12C). The observation of only one signal for BAC $13 C 5$ indicates that the duplication event is confined to a region of $13 \mathrm{q} 31$ that is distal to $\mathrm{BAC} 13 \mathrm{C} 5$. The orientation of the duplication was delineated as an inversion duplication using dual color FISH with BACs $13 \mathrm{C} 5 / 13 \mathrm{C} 7$ (data not shown); 13C5/13C11 and $13 \mathrm{C} 7 / 13 \mathrm{C} 11$ which showed signals in the order of (proximal to distal) BAC $13 \mathrm{C} 5, \mathrm{BAC} 13 \mathrm{C} 7, \mathrm{BAC}$ 13C11, BAC 13C11, BAC 13C7 (Fig. 1:12C).

\section{DISCUSSION}

The rapid advance of molecular cytogenetic technology has led to an increased number of referrals of specimens to specialized laboratories from patients with complex and unresolvable G-banded karyotypes. In clinical cytogenetics, the precise identification of the origin of the additional or missing chromosomal material is a key factor when considering genotype-phenotype correlations and ultimately may lead to the discovery of the genes responsible for the clinical abnormalities that present in such patients. Defining the origin of unknown additional cytogenetic material with FISH by use of various probes is expensive and laborious because many whole chromosome paints may be required until the source chromosome is identified. In the present series, 6 of the 12 cases were subjected to multiple WCPs and locus-specific probes by the referring institutions before being sent to our laboratory for CGH analysis. The identification of additional undefinable cytogenetic material can also be achieved using other molecular cytogenetic techniques like reverse FISH ${ }^{6-8}$ and multicolor FISH (spectral karyotyping and M-FISH). ${ }^{9,10}$ Although the information derived using reverse FISH is highly informative, the procedure is technically demanding and requires specialized micromanipulation equipment to microdissect and prepare probes from the region of interest. CGH is an alternative technique that can characterize unbalanced, unrecognizable G-banded cytogenetic material in a one-step global screening procedure. The advantage of CGH over multicolor FISH is its ability to identify not only the chromosome from which the additional unknown material was derived but to map the region involved to specific bands on the source chromosome. Although multicolor FISH would be able to identify intrachromosomal duplications such as those in cases 11 and 12 , it would not be possible to characterize the involved regions with the same resolution obtained by CGH. Multicolor FISH has proven to be a powerful tool in the cytogenetic analysis of solid tumors. However, its success depends on the presence and to some extent the quality of metaphase spreads. This requirement limits its usefulness in cancer research and clinical cytogenetics where the lack of metaphases is quite common in certain solid tissue samples such as tumors and abortus material.

In the past, DA-DAPI staining has proved effective in identifying extrachromosomal material derived from proximal chromosome 15 . Because $80 \%$ of markers originate from acrocentric chromosomes and half of these are derived from chromosome $15,{ }^{26}$, ${ }^{27}$ the sole use of DA-DAPI staining to characterize the marker derived from chromosome 1 in case 5 may have led to the mistaken conclusion that it was derived from chromosome 15 . This is because DADAPI staining highlights the heterochromatic regions of chromosomes $1,9,16$, and $\mathrm{Yqh}$ in addition to 15pl1 (see Fig. 1: 1C and 2C). Chromosome 15 markers have been characterized extensively $y^{28-32}$ and isochromosome $18 \mathrm{p}$ markers have also been well documented. ${ }^{29,33-35}$ Chromosome 1 markers are certainly rare but their presence may be under-reported especially in the premolecular era when DA-DAPI staining, in the absence of additional staining such as C-banding or NOR-banding, may have led to the labeling of such markers as being derived from chromosome 15 . The number of reported clinical cases involving chromosome 1 markers is limited to 7, as summarized recently by Crolla. ${ }^{27}$

To date, more than 180 articles have been published on $\mathrm{CGH}$ with the majority (approximately $80 \%$ ) reporting the utility of $\mathrm{CGH}$ in the delineation of cytogenetic changes in more than 1500 tumors. ${ }^{36}$ The remaining $\mathrm{CGH}$ papers have dealt primarily with technical aspects and only a few have described the application of CGH in clinical cytogenetics. ${ }^{15-22}$ $\mathrm{CGH}$ analysis software is now readily available as an optional addition to many FISH imaging systems and does not require additional specialized equipment. With many comprehensive cytogenetic laboratories now acquiring image analysis equipment for routine cytogenetic and FISH analysis, the use of $\mathrm{CGH}$ as a tool in clinical cytogenetics is likely to increase. In addition to being able to identify excess and/or missing chromosomal material not resolvable by G-banding, CGH could also be used as a backup method for aneuploidy analysis of specimens that have failed to grow in cell culture. This would be particularly useful in the analysis of nonviable fetal tissue derived from products of conception that is estimated to have a chromosome abnormality (mainly aneuploidy) approximately $50 \%$ of the time.

The sensitivity of CGH is an issue that has received much attention and, to date, no intensive and comprehensive study has been undertaken to accurately establish the minimum size of chromosome imbalance that $\mathrm{CGH}$ can detect. The theoretical detection limit of deletions by CGH has been estimated to be about $2 \mathrm{Mb}^{37}$ Bentz et al. ${ }^{38}$ recently reported 10 to $12 \mathrm{Mb}$ to be the minimal size of deletions that $\mathrm{CGH}$ could detect. Their study samples, however, consisted of only five cases which were all mosaic for $11 \mathrm{q}$ deletions. Whether the sensitivity would have improved if the samples were nonmosaic was not addressed by those researchers. The technique of CGH is itself quite demanding and certainly requires considerable experience to obtain optimal results. Ghaffari et al. ${ }^{22}$ recently used a modified $\mathrm{CGH}$ analysis strategy for identifying cryptic telomeric translocations in patients with idiopathic mental retardation. They were able to identify deletions and duplication as small as $4 \mathrm{Mb}$ and $5 \mathrm{Mb}$, respectively. In contrast, Griffin et al. ${ }^{21}$ were unable to use regular CGH to determine the origin of intrachromosomal duplications that were less than $10 \mathrm{Mb}$. They achieved increased CGH resolution by performing chromosome-specific CGH that combines flow sorting of chromosomes, degenerate oligonucleotide primed 
(DOP)-PCR, and a modified CGH technique. We were recently able to identify an intrachromosomal duplication in the range of $7 \mathrm{Mb}$ (data not included in this study) using a standard CGH protocol. Whole chromosome aneuploidy clearly is within the detection limits of $\mathrm{CGH}$. The ability of $\mathrm{CGH}$ to detect partial aneuploidy in the form of marker chromosomes, unbalanced translocations, and intrachromosomal duplications is demonstrated effectively in this communication. CGH easily identified the additional material on all chromosomes in question in this report. In the unbalanced translocation cases in which the reciprocal monosomic regions were not evident in the $\mathrm{CGH}$ ratio profiles, the breakpoints could be designated from the GTG image as the terminal band of the derivative chromosome.

In conclusion, the use of CGH is rapidly being expanded in a clinical cytogenetic setting. As technical advances improve the capabilities of $\mathrm{CGH}$, future additional applications are likely to include screening for deletions as small as those observed in the Prader-Willi and DiGeorge microdeletion syndromes. This will be made possible by the recent development of a matrix CGH protocol that combines biochip and $\mathrm{CGH}$ technologies. ${ }^{39}$ Using regular CGH would still remain an attractive and powerful accessory to routine clinical cytogenetic analysis.

\section{Acknowledgments}

We are deeply indebted to Drs. Carpenter, Fallet, Fox, Lieber, Shapiro, and Zackai for referring their clinical cases to us for investigation. Without their assistance, this report would not have been possible. This work was supported in part by grants from the National Institutes of Health (5 T32 07105) and Lehman Brothers, Inc.

\section{References}

1. Harper PS, editor. Practical genetic counseling, 4th ed. Oxford: Butterworth-Heinemann Ltd, 1993.

2. Gardner RJM, Sutherland GR, editors. Chromosome abnormalities and genetic counseling, 2nd ed. New York: Oxford University Press, 1996.

3. Leana-Cox J, Levin S, Surana R, Wulfsberg E, Keene CL, Raffel $L J$, et al. Characterization of de novo duplications in eight patients using fluorescence in situ hybridization with chromosome-specific DNA libraries. Am I Hum Genet 1993;52:1067-1073.

4. Blennow E, Bui TH, Kristoffersson U, Vujic M, Annerén G, Holmberg $E$, et al. Swedish survey on extra structurally abnormal chromosomes in 39,105 consecutive prenatal diagnoses: Prevalence and characterization by fluorescence in situ hybridization. Prenat Diagn 1994;14:1019-1028.

5. Blennow E, Brondum K, Håkan T, Carter NP, Kristoffersson $U$, Holmberg E, et al. Fifty probands with extra structurally abnormal chromosomes characterized by fluorescence in situ hybridization. Am J Med Genet 1995;55:85-94.

6. Ohta T, Tohma T, Soejima H, Fukushima Y, Nagai T, Yoshiura $\mathrm{K}$, et al. The origin of cytologically unidentifiable chromosome abnormalities: Six cases ascertained by targeted chromosome-band painting. Hum Genet 1993;92:1-5.

7. Thangavelu M, Pergament E, Espinosa R, Bohlander SK. Characterization of marker chromosomes by microdissection and fluorescence in situ hybridization. Prenat Diagn 1994;14:583-588.

8. Viersbach R, Schwanitz G, Nöthen MM. Delineation of marker chromosomes by reverse chromosome painting using only a small number of DOP-PCR amplified microdissected chromosomes. Hum Genet 1994;93:663-667.

9. Schröck E, Du Manoir S, Veldman T, Schoell B, Weinberg J, Ferguson-Smith MA, et al. Multicolor spectral karyotyping of human chromosomes. Science 1996;273:494-497.

10. Speicher MR, Ballard SG, Ward DC. Karyotyping human chromosomes by combinatorial multi-fluor FISH. Nat Genet 1996;12:368-375.

11. Kallioniemi A, Kallioniemi OP, Sudar D, Rutovitz D, Gray IW, Waldman F, et al. Comparative genomic hybridization for molecular cytogenetic analysis of solid tumors. Science 1992;258:818-821.

12. Kallioniemi OP, Kallioniemi A, Sudar D, Rutovitz D, Gray JW, Waldman F, et al. Comparative genomic hybridization: $A$ rapid new method for detecting and mapping DNA amplification in tumors. Semin Cancer Biol 1993:4:41-46.

13. Du Manoir S, Speicher MR, Joos S, Schröck E, Popp S, Döhner $\mathrm{H}$, et al. Detection of complete and partial chromosome gains and losses by comparative genomic in situ hybridization. Hum Genet 1993;90:590-610.

14. Kallioniemi OP, Kallioniemi A, Piper J, Isola J, Waldman FM, Gray JW, et al. Optimizing comparative genomic hybridization for analysis of DNA sequence copy number changes in solid tumors. Genes Chromosomes Cancer 1994;10:231-243.

15. Bryndorf T, Kirchhoff M, Rose H, Maahr J, Gerdes T, Karhu $\mathrm{R}$, et al. Comparative genomic hybridization in clinical cytogenetics. Am J Hum Genet 1995;57:1211-1220.

16. Wang BB, Yu L, Willow P, Falk RE, Williams J. Prenatal identification of $\mathrm{i}(\mathrm{Yp})$ by molecular cytogenetic analysis. Prenat Diagn 1995;15:1115-1119.

17. Wegner RD, Schröck E, Obladen M, Becker R, Stumm M, Sperling K. Partial trisomy/monosomy $6 q$ in fetal cells and CVS long-term culture not present in CVS short-term culture. Prenat Diagn 1996;16:741-748.

18. Levy B, Gershin IF, Desnick RJ, Babu A, Gelb BD, Hirschhorn $\mathrm{K}$, et al. Characterization of a de novo unbalanced chromosome rearrangement by comparative genomic hybridization and fluorescence in situ hybridization. Cytogenet Cell Genet 1997;76:68-71.

19. Erdel M, Duba HC, Verdorfer I, Lingenhel A, Geiger R, Gutenberger $\mathrm{KH}$, et al. Comparative genomic hybridization reveals a partial de novo trisomy 6q23-qter in an infant with congenital malformations: Delineation of the phenotype. Hum Genet 1997;99:596-601.

20. Browne CE, Dennis NR, Maher E, Long FL, Nicholson JC, Sillibourne J, et al. Inherited interstitial duplications of proximal 15q: Genotype-phenotype correlations. Am J Hum Genet 1997;61:1342-1352.

21. Griffin DK, Sanoudou D, Adamski E, McGiffert C, O'Brien $\mathrm{P}$, Wienberg J, et al. Chromosome specific comparative genome hybridization for determining the origin of intrachromosomal duplications. J Med Genet 1998;35:37-41.

22. Ghaffari SR, Boyd E, Tolmie JL, Crow YJ, Trainer AH, Connor JM. A new strategy for cryptic telomeric translocation screening in patients with idiopathic mental retardation. JMed Genet 1998:35:225-233.

23. Sambrook J, Fritsch EF, Maniatis T. Molecular cloning: A laboratory manual, 2nd ed. Cold Spring Harbor, NY: Cold Spring Harbor Press, 1989.

24. Florijn RJ, Slats I, Tanke HJ, Raap AK. Analysis of antifading reagents for fluorescence microscopy. Cytometry 1995;19:177-182.

25. Du Manoir S, Kallioniemi OP, Lichter P, Piper J, Benedetti PA, Carothers $\mathrm{AD}$, et al. Hardware and software requirements for quantitative analysis of comparative genomic hybridization. Cytometry 1995;19:4-9.

26. Crolla JA, Long F, Rivera H, Dennis NR. FISH and molecular study of autosomal supernumerary marker chromosomes excluding those derived from chromosomes 15 and 22 . I. Results from 26 new cases. Am J Med Genet 1998;75:355-366.

27. Crolla JA. FISH and molecular studies of autosomal supernumerary marker chromosomes excluding those derived from chromosomes 15. Il. Review of the literature. Am J Med Genet 1998;75:367-381.

28. Wisniewski LP, Witt ME, Ginsberg-Fellner F, Wilner J, Desnick RJ. Prader-Willi syndrome and a bisatellited derivative of chromosome 15. Clin Genet 1980;18:42-47.

29. Schinzel A, editor. Catalogue of unbalanced chromosome aberrations in man. Berlin: Walter de Gruyter, 1984.

30. Maraschio P, Cuoco C, Gimelli G, Zuffardi O, Tiepolo L. 
Origin and clinical significance of inv dup(15). In: Daniel A, editor. The cytogenetics of mammalian autosomal rearrangements. New York: Alan R. Liss, 1988;615.

31. Leana-Cox J, Jenkins L, Palmer CG, Plattner R, Sheppard L, Flejter WL, et al. Molecular cytogenetic analysis of inv dup(15) chromosomes using probes specific for the PraderWilli/Angelman syndrome region: Clinical implications. Am J Hum Genet 1994;54:748-756.

32. Webb T, Hardy CA, King M, Watkiss E, Mitchell C, Cole T, et al. A clinical, cytogenetic and molecular study of ten probands with supernumerary inv dup (15) marker chromosomes. Clin Genet 1998;53:34-43.

33. Condron CJ, Cantwell RJ, Kaufman RL, Brown SB, Warren $\mathrm{RJ}$. The supernumerary isochromosome 18 syndrome (+18pi). Birth Defects Orig Artic Ser 1974;10:36-42.

34. Rocchi M, Stormi M, Archidiacono N, Filippi G. Extra small metacentric chromosome identified as i(18p). J Med Genet 1979;16:69-73.
35. Darnaude MT, Diaz de Bustamante A, Cabello P, Vallcorba I. Genetic counselling in a prenatal marker chromosome identified as an i (18p) by in situ hybridization. Ann Genet 1996;39:61-63.

36. Forozan F, Karhu R, Kononen I, Kallioniemi A, Kallioniemi $O P$. Genome screening by comparative genomic hybridization. Trends Genet 1997;13:405-409.

37. Piper I, Rutovitz D, Sudar D, Kallioniemi A, Kallioniemi OP, Waldman FM, et al. Computer image analysis of comparative genomic hybridization. Cytometry 1995;19:10-26.

38. Bentz M, Plesch A, Stilgenbauer S, Döhner $H$, Lichter P. Minimal sizes of deletions detected by comparative genomic hybridization. Genes Chromosomes Cancer 1998;21:172-175.

39. Solinas-Toldo S, Lampel S, Stilgenbauer S, Nickolenko I, Benner A, Döhner $\mathrm{H}$, et al. Matrix-based comparative genomic hybridization: Biochips to screen for genomic imbalances. Genes Chromosomes Cancer 1997;20:399-407. 